Supplement of Atmos. Chem. Phys., 19, 7335-7345, 2019

https://doi.org/10.5194/acp-19-7335-2019-supplement

(C) Author(s) 2019. This work is distributed under

the Creative Commons Attribution 4.0 License.

(c) (1)

Supplement of

\title{
Temporal variations and trend of ground-level ozone based on long-term measurements in Windsor, Canada
}

Xiaohong Xu et al.

Correspondence to: Xiaohong Xu (xxu@uwindsor.ca)

The copyright of individual parts of the supplement might differ from the CC BY 4.0 License. 


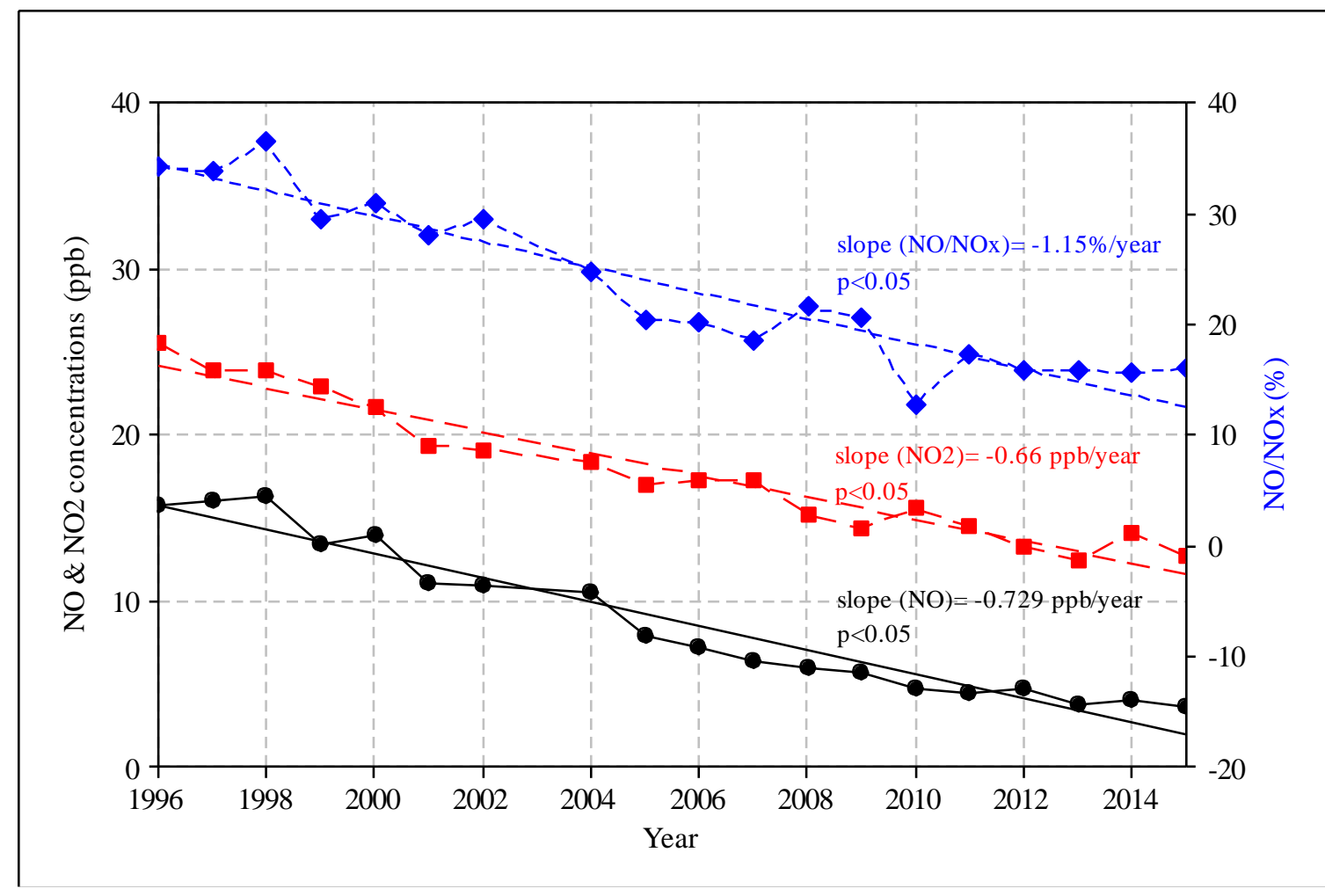

Figure S1. Long-term trends of $\mathrm{NO}, \mathrm{NO}_{2}$, and $\mathrm{NO} / \mathrm{NO}_{\mathrm{X}}$ in Windsor during 1996-2015. 

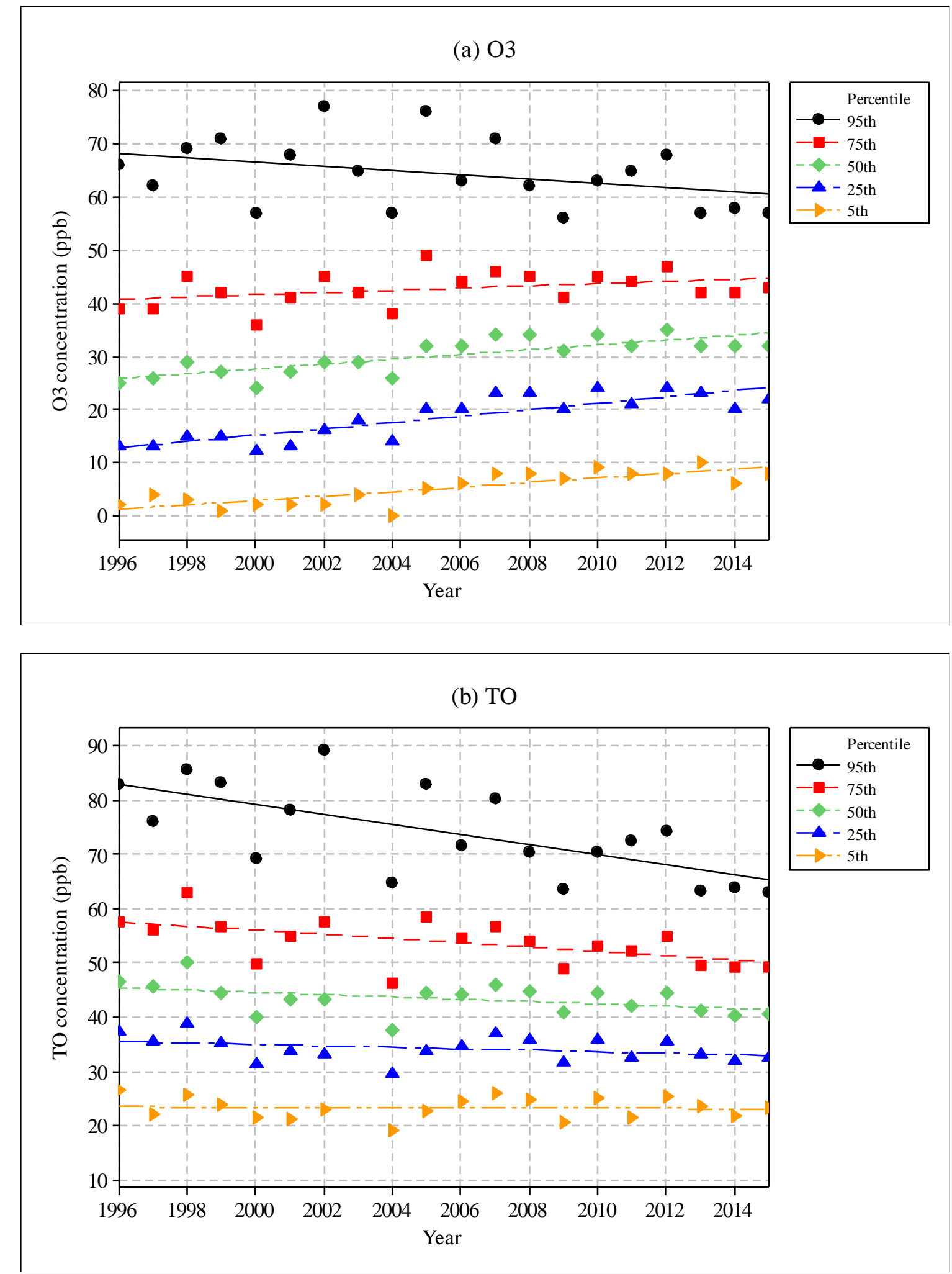

Figure S2. Long-term trends at various percentile levels in smog season in Windsor, a) $\mathrm{O}_{3}$, and b) TO. 


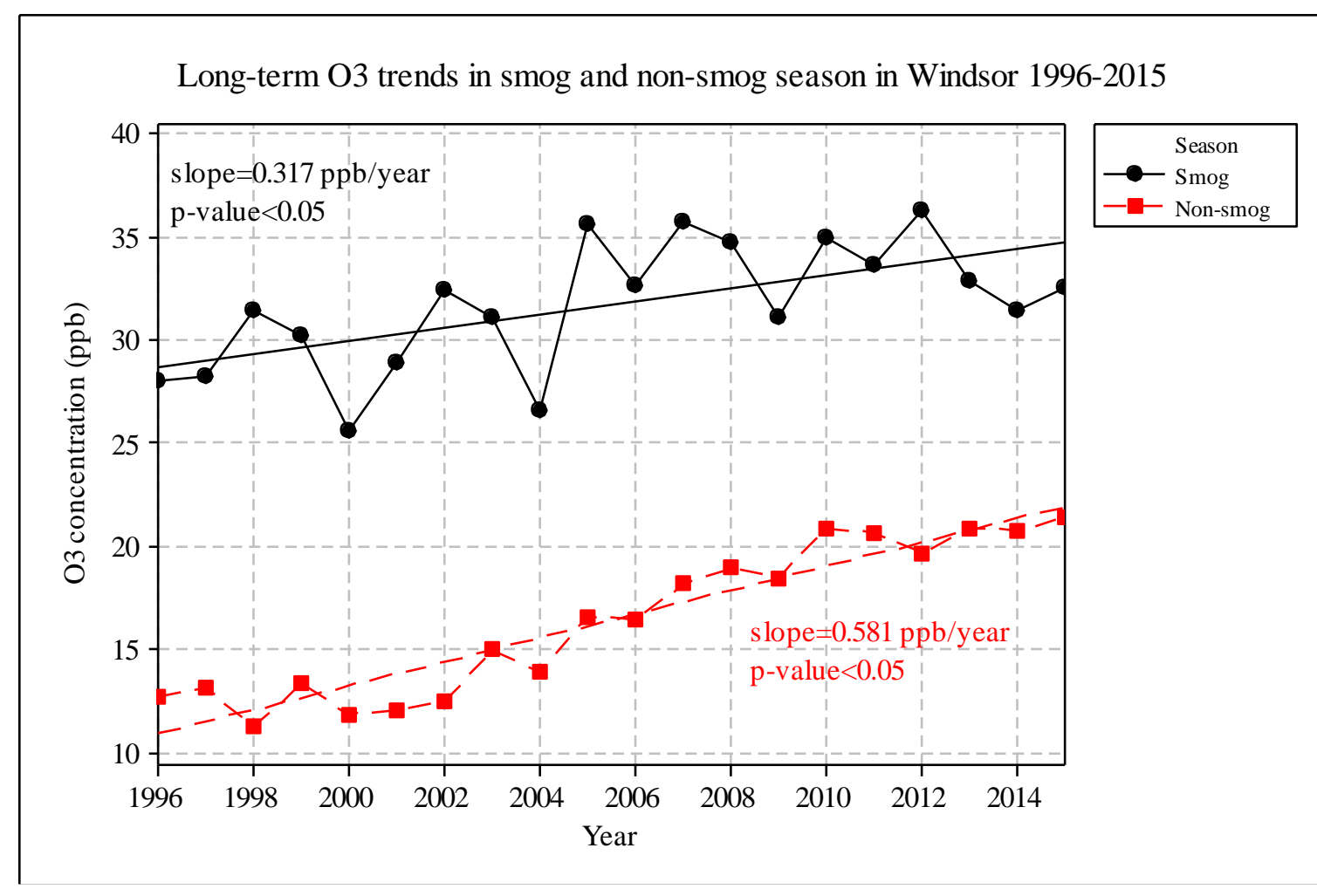

Figure S3. Long-term $\mathrm{O}_{3}$ trends in smog and non-smog season in Windsor 1996-2015. 
Table S1. Numbers of invalid (flagged) or blank cells, and percentage of valid data in each year during 1996-2015 in Windsor for $\mathrm{O}_{3}, \mathrm{NO}, \mathrm{NO}_{2}$, and $\mathrm{NO}_{\mathrm{x}}$.

\begin{tabular}{|c|c|c|c|c|c|c|c|c|c|c|c|c|}
\hline \multirow[b]{2}{*}{ Year } & \multicolumn{3}{|c|}{$\mathrm{O}_{3}$} & \multicolumn{3}{|c|}{$\mathrm{NO}$} & \multicolumn{3}{|c|}{$\mathrm{NO}_{2}$} & \multicolumn{3}{|c|}{$\mathrm{NO}_{\mathrm{X}}$} \\
\hline & $\begin{array}{l}\text { \# of } \\
\text { flag }\end{array}$ & $\begin{array}{c}\text { \# of } \\
\text { blank }\end{array}$ & $\begin{array}{c}\% \\
\text { valid }\end{array}$ & $\begin{array}{l}\text { \# of } \\
\text { flag }\end{array}$ & $\begin{array}{c}\text { \# of } \\
\text { blank }\end{array}$ & $\begin{array}{c}\% \\
\text { valid }\end{array}$ & $\begin{array}{l}\text { \# of } \\
\text { flag }\end{array}$ & $\begin{array}{c}\text { \# of } \\
\text { blank }\end{array}$ & $\begin{array}{c}\% \\
\text { valid }\end{array}$ & $\begin{array}{l}\text { \# of } \\
\text { flag }\end{array}$ & $\begin{array}{c}\text { \# of } \\
\text { blank }\end{array}$ & $\begin{array}{c}\% \\
\text { valid }\end{array}$ \\
\hline 1996 & 9 & 53 & 99.3 & 12 & 44 & 99.4 & 9 & 53 & 99.3 & 6 & 58 & 99.3 \\
\hline 1997 & 1 & 150 & 98.3 & 170 & 145 & 96.4 & 170 & 145 & 96.4 & 117 & 142 & 97.0 \\
\hline 1998 & 1 & 89 & 99.0 & 87 & 24 & 98.7 & 87 & 24 & 98.7 & 87 & 24 & 98.7 \\
\hline 1999 & 97 & 59 & 98.2 & 38 & 214 & 97.1 & 35 & 119 & 98.2 & 36 & 231 & 97.0 \\
\hline 2000 & 98 & 23 & 98.6 & 209 & 23 & 97.4 & 209 & 23 & 97.4 & 209 & 23 & 97.4 \\
\hline 2001 & 71 & 1 & 99.2 & 170 & 1 & 98.0 & 170 & 1 & 98.0 & 170 & 1 & 98.0 \\
\hline 2002 & 153 & 1308 & 83.3 & 167 & 1308 & 83.2 & 167 & 1308 & 83.2 & 167 & 1308 & 83.2 \\
\hline 2003 & 235 & 1 & 97.3 & 5320 & 25 & 39.0 & 5320 & 25 & 39.0 & 5343 & 2 & 39.0 \\
\hline 2004 & 38 & 1 & 99.6 & 220 & 1 & 97.5 & 220 & 1 & 97.5 & 220 & 1 & 97.5 \\
\hline 2005 & 748 & 7 & 91.4 & 205 & 7 & 97.6 & 199 & 7 & 97.6 & 199 & 7 & 97.6 \\
\hline 2006 & 18 & 5 & 99.7 & 24 & 5 & 99.7 & 24 & 5 & 99.7 & 24 & 5 & 99.7 \\
\hline 2007 & 48 & 3 & 99.4 & 14 & 3 & 99.8 & 14 & 3 & 99.8 & 14 & 3 & 99.8 \\
\hline 2008 & 10 & 62 & 99.2 & 19 & 62 & 99.1 & 19 & 62 & 99.1 & 19 & 62 & 99.1 \\
\hline 2009 & 48 & 1 & 99.4 & 316 & 1 & 96.4 & 316 & 1 & 96.4 & 316 & 1 & 96.4 \\
\hline 2010 & 23 & 4 & 99.7 & 186 & 4 & 97.8 & 186 & 4 & 97.8 & 186 & 4 & 97.8 \\
\hline 2011 & 26 & 0 & 99.7 & 20 & 0 & 99.8 & 20 & 0 & 99.8 & 20 & 0 & 99.8 \\
\hline 2012 & 41 & 2 & 99.5 & 18 & 2 & 99.8 & 18 & 2 & 99.8 & 18 & 2 & 99.8 \\
\hline 2013 & 23 & 15 & 99.6 & 39 & 15 & 99.4 & 39 & 15 & 99.4 & 39 & 15 & 99.4 \\
\hline 2014 & 153 & 39 & 97.8 & 36 & 39 & 99.1 & 36 & 39 & 99.1 & 36 & 39 & 99.1 \\
\hline 2015 & 31 & 1 & 99.6 & 14 & 1 & 99.8 & 14 & 1 & 99.8 & 14 & 1 & 99.8 \\
\hline
\end{tabular}


Table S2. Hour-of-day TO, $\mathrm{O}_{3}$, and $\mathrm{DO}_{3}$ in Windsor during 1996-2015, a) concentrations, and b) slopes. (in each column, darker colors indicate larger values)

a) Concentrations

\begin{tabular}{cccc}
\hline Hour & $\begin{array}{c}\mathrm{TO} \\
(\mathrm{ppb})\end{array}$ & $\begin{array}{c}\mathrm{O}_{3} \\
(\mathrm{ppb})\end{array}$ & $\begin{array}{c}\mathrm{DO}_{3} \\
(\mathrm{ppb})\end{array}$ \\
\hline 0 & 36.6 & 19.6 & 16.8 \\
1 & 35.3 & 18.7 & 16.5 \\
2 & 34.5 & 18.2 & 16.2 \\
3 & 33.7 & 17.5 & 16.0 \\
4 & 32.9 & 16.4 & 16.4 \\
5 & 32.1 & 14.5 & 17.5 \\
6 & 31.9 & 13.3 & 18.5 \\
7 & 32.7 & 14.1 & 18.5 \\
8 & 34.4 & 17.4 & 16.9 \\
9 & 36.6 & 21.8 & 14.7 \\
10 & 39.1 & 26.1 & 12.9 \\
11 & 41.6 & 29.9 & 11.6 \\
12 & 43.8 & 32.8 & 10.9 \\
13 & 45.5 & 34.7 & 10.8 \\
14 & 46.5 & 35.5 & 11.0 \\
15 & 47.0 & 35.3 & 11.6 \\
16 & 46.7 & 34.2 & 12.5 \\
17 & 46.1 & 32.4 & 13.5 \\
18 & 44.8 & 30.0 & 14.6 \\
19 & 43.1 & 27.1 & 15.9 \\
20 & 41.3 & 24.2 & 17.0 \\
21 & 39.8 & 22.2 & 17.5 \\
22 & 38.5 & 20.8 & 17.6 \\
23 & 37.5 & 20.1 & 17.3 \\
\hline & & &
\end{tabular}

b) Slopes

\begin{tabular}{cccc}
\hline Hour & $\begin{array}{c}\mathrm{TO} \\
(\mathrm{ppb} / \text { year })\end{array}$ & $\begin{array}{c}\mathrm{O}_{3} \\
(\mathrm{ppb} / \text { year })\end{array}$ & $\begin{array}{c}\mathrm{DO}_{3} \\
(\mathrm{ppb} / \text { year }\end{array}$ \\
\hline 0 & 0.01 & 0.62 & -0.59 \\
1 & -0.01 & 0.55 & -0.55 \\
2 & -0.01 & 0.50 & -0.51 \\
3 & -0.01 & 0.50 & -0.48 \\
4 & 0.00 & 0.44 & -0.45 \\
5 & 0.00 & 0.42 & -0.44 \\
6 & 0.03 & 0.40 & -0.44 \\
7 & 0.05 & 0.45 & -0.46 \\
8 & 0.05 & 0.50 & -0.46 \\
9 & 0.05 & 0.50 & -0.45 \\
10 & 0.05 & 0.50 & -0.43 \\
11 & 0.03 & 0.50 & -0.42 \\
12 & 0.01 & 0.44 & -0.41 \\
13 & -0.02 & 0.40 & -0.41 \\
14 & -0.02 & 0.42 & -0.43 \\
15 & -0.02 & 0.46 & -0.46 \\
16 & -0.01 & 0.50 & -0.50 \\
17 & -0.02 & 0.53 & -0.53 \\
18 & -0.02 & 0.57 & -0.56 \\
19 & 0.00 & 0.60 & -0.58 \\
20 & 0.00 & 0.63 & -0.62 \\
21 & 0.00 & 0.64 & -0.63 \\
22 & 0.00 & 0.67 & -0.65 \\
23 & 0.00 & 0.64 & -0.63 \\
\hline & & & \\
\hline
\end{tabular}


Table S3. Month-of-year TO, $\mathrm{O}_{3}$, and $\mathrm{DO}_{3}$ in Windsor during 1996-2015, a) concentrations, and b) slopes. (in each column, darker colors indicate larger values)

a) Concentrations

\begin{tabular}{cccc}
\hline Month & $\begin{array}{c}\mathrm{TO} \\
(\mathrm{ppb})\end{array}$ & $\begin{array}{c}\mathrm{O}_{3} \\
(\mathrm{ppb})\end{array}$ & $\begin{array}{c}\mathrm{DO}_{3} \\
(\mathrm{ppb})\end{array}$ \\
\hline 1 & 32.4 & 13.7 & 18.8 \\
2 & 37.2 & 18.3 & 18.7 \\
3 & 42.0 & 25.2 & 16.5 \\
4 & 45.1 & 30.3 & 14.5 \\
5 & 45.7 & 32.3 & 13.3 \\
6 & 48.5 & 35.3 & 13.1 \\
7 & 47.5 & 35.1 & 12.5 \\
8 & 44.0 & 31.2 & 12.9 \\
9 & 39.6 & 25.9 & 13.6 \\
10 & 31.7 & 17.0 & 14.7 \\
11 & 29.5 & 13.3 & 16.1 \\
12 & 28.6 & 11.5 & 17.2 \\
\hline
\end{tabular}

b) Slopes

\begin{tabular}{cccc}
\hline Month & $\begin{array}{c}\mathrm{TO} \\
(\mathrm{ppb} / \text { year })\end{array}$ & $\begin{array}{c}\mathrm{O}_{3} \\
(\mathrm{ppb} / \text { year })\end{array}$ & $\begin{array}{c}\mathrm{DO}_{3} \\
(\mathrm{ppb} / \text { year })\end{array}$ \\
\hline 1 & 0.28 & 0.63 & -0.37 \\
2 & 0.43 & 0.77 & -0.36 \\
3 & 0.15 & 0.58 & -0.43 \\
4 & -0.10 & 0.60 & -0.57 \\
5 & -0.18 & 0.46 & -0.50 \\
6 & -0.39 & 0.27 & -0.53 \\
7 & -0.24 & 0.33 & -0.45 \\
8 & -0.21 & 0.36 & -0.47 \\
9 & -0.34 & 0.35 & -0.57 \\
10 & -0.17 & 0.40 & -0.53 \\
11 & 0.14 & 0.60 & -0.48 \\
12 & 0.16 & 0.54 & -0.45 \\
\hline
\end{tabular}

Table S4. Slopes of long-term $\mathrm{O}_{3}$ and TO trends at various percentile levels in all months, the smog season, and the non-smog season in Windsor during 1996-2015.

\begin{tabular}{ccccccc}
\hline \multicolumn{2}{c}{ Slope (ppb/year) } & 5 th & 25 th & 50 th & 75 th & 95 th \\
\hline \multirow{2}{*}{$\mathrm{O}_{3}$} & All months & $0.171^{* *}$ & $0.731^{* *}$ & $0.635^{* *}$ & $0.42^{* *}$ & -0.163 \\
& Smog & $0.395^{* *}$ & $0.548^{* *}$ & $0.395^{* *}$ & 0.148 & $-0.447^{*}$ \\
& Non-smog & $0.124^{* *}$ & $0.723^{* *}$ & $0.771^{* *}$ & $0.631^{* *}$ & $0.467 * *$ \\
\multirow{2}{*}{$\mathrm{TO}$} & All months & 0.119 & 0.101 & 0.0751 & $-0.149 *$ & $-0.736^{* *}$ \\
& Smog & -0.022 & $-0.158^{*}$ & $-0.276^{* *}$ & $-0.455^{* *}$ & $-0.995^{* *}$ \\
& Non-smog & 0.169 & $0.186^{* *}$ & $0.219^{* *}$ & 0.144 & -0.125 \\
\hline
\end{tabular}

* significant at $\mathrm{p}<0.1 ; * *$ significant at $\mathrm{p}<0.05$ 